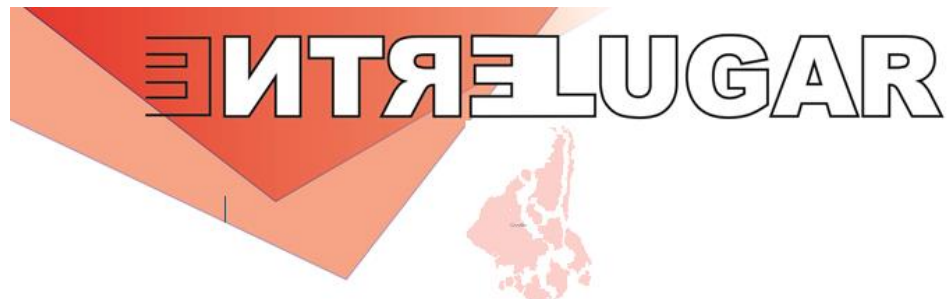

\title{
CHUVAS EM UBERABA/MG: UM ESTUDO SOBRE A OCORRÊNCIA DE EVENTOS EXTREMOS
}

\author{
RAINFALL IN UBERABA/MG: A STUDY ON THE OCCURRENCE OF \\ EXTREME EVENTS
}

\section{LLUVIAS EN UBERABA / MG: UN ESTUDIO SOBRE LA OCURRENCIA DE EVENTOS EXTREMOS}

Cléo Maycon Viana Paz

cleomaycon.vianapaz@hotmail.com Universidade Federal do Triangulo Mineiro

Fabio de Oliveira Sanches

E-mail: fsanches.73@gmail.com Universidade Federal de Juiz de Fora

Ricardo Vicente Ferreira

E-mail: rcrdvf@gmail.com Universidade Federal do Triangulo Mineiro

Resumo: O presente trabalho ocupou-se com o estudo do comportamento das chuvas em Uberaba/MG, identificando os eventos extremos de precipitação, sua frequência e seu tempo de retorno. Foram utilizados dados diários da Estação Meteorológica do INMET de Uberaba, no período de 1961-2015. O Teste de Mann-Kendall foi aplicado aos totais mensais e aos parâmetros propostos pelo ETCCDI para a pluviosidade. A aplicação do percentil 99 aos dados pluviométricos diários permitiu a identificação dos eventos extremos. Foram calculados a frequência desses eventos (f), a frequência relativa (probabilidade de ocorrência) e o Tempo de retorno (T) desses eventos. Os resultados revelaram que há uma tendência significativa de aumento da precipitação no mês de março, da quantidade de dias de chuva e de eventos intensos de precipitação diária. Por outro lado, as chuvas no mês de outubro apresentaram tendência de redução significativa, bem como a diminuição dos dias de chuva para este mês. Os resultados ainda demonstraram que as chuvas diárias intensas são muito comuns em Uberaba ocorrendo, pelo menos, dois eventos significativos a cada ano.

Palavras-chave: mudanças climáticas, precipitações, testes estatísticos, Triângulo Mineiro.

Abstract: The present work was concerned with the study of rain behavior in Uberaba / MG, identifying the extreme precipitation events, their frequency and their return time. 


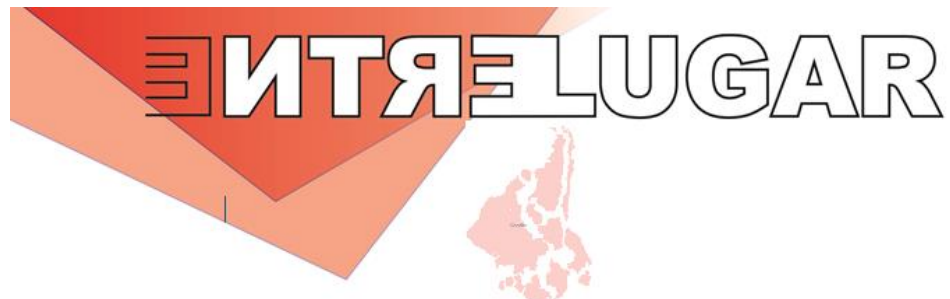

Daily data from 1961 to 2015 period, from the INMET Meteorological Station of Uberaba were analyzed. The Mann-Kendall test was applied to the monthly totals and parameters proposed by the ETCCDI for rainfall. The application of the 99th percentile to the daily rainfall data identified extreme events. The frequency of these events (f), the relative frequency (probability of occurrence) and the time of return (t) of these events were calculated. Results showed a significant tendency of rainfall increase in the month of March, the amount of rainy days and intense daily precipitation events. On the other hand, rainfall in October showed a significant reduction trend, as well as a decrease in rainy days for this month. The results also demonstrated that intense daily rains are very common in Uberaba, occurring at least two significant events each year.

Keywords: climate change, rainfall, statistical tests, Triângulo Mineiro Region.

Resumen: El presente trabajo se ocupó del estudio del comportamiento de la lluvia en Uberaba / MG, identificando los eventos extremos de precipitación, su frecuencia y su tiempo de retorno. El objetivo de esta investigación fue evaluar el comportamiento local de la lluvia en relación con los cambios climáticos, identificando los eventos extremos de precipitación, su frecuencia y período de retorno. Se analizaron datos diarios del período 1961 a 2015, de la Estación Meteorológica INMET de Uberaba. La prueba de MannKendall se aplicó a los totales y parámetros mensuales propuestos por el ETCCDI para la lluvia. La aplicación del percentil 99 a los datos de lluvia diaria identificó eventos extremos. Se calcularon la frecuencia de estos eventos (f), la frecuencia relativa (probabilidad de ocurrencia) y el tiempo de retorno (t) de estos eventos. Los resultados mostraron una tendencia significativa al aumento de las precipitaciones en el mes de marzo, la cantidad de días lluviosos y los intensos eventos de precipitación diaria. Por otro lado, la lluvia en octubre mostró una tendencia de reducción significativa, así como una disminución en los días lluviosos para este mes. Los resultados también demostraron que las intensas lluvias diarias son muy comunes en Uberaba, y ocurren al menos dos eventos significativos cada año.

Palabras clave: cambio climático, lluvia, pruebas estadísticas, Triângulo Mineiro.

\section{INTRODUÇÃOO}

O clima é uma componente da natureza que condiciona o modo de vida das sociedades desde os primórdios da humanidade. Antigas civilizações que viveram na região da Mesopotâmia e Egito antigo já possuíam conhecimento climático relacionado à sazonalidade das chuvas ao longo de um ano (MENDONÇA \& DANNI-OLIVEIRA, 2007). Com o passar do tempo, o conhecimento sistematizado pela ciência (técnica, método e tecnologia), possibilitou que a sociedade compreendesse melhor as questões relacionadas à sazonalidade, variabilidade e características do clima no planeta. 
No entanto, mesmo com os maiores avanços científicos na área, a sociedade ainda é muito vulnerável aos fenômenos naturais e, diante do cenário atual, as mudanças climáticas globais e os eventos extremos oriundos delas, sobretudo a partir da segunda metade do século XX, têm sido responsáveis por acentuar parte dos problemas sociais, econômicos e ambientais nas escalas global, regional e local.

O Painel Intergovernamental de Mudanças Climática (IPCC, sigla em inglês) considera que os eventos extremos são grandes variações que afetam a normal climatológica de um determinado lugar, de forma que o comportamento climático em dada ocasião seja muito distinto das médias de temperatura e pluviosidade comuns.

Neste sentido, as chuvas intensas em um curto período de tempo podem provocar enchentes, deslizamentos de terra e movimentos de massa. Estes são alguns dos problemas apontados pelo $5^{\circ}$ relatório (AR5) do IPCC (IPCC, 2014) em relação às mudanças climáticas em cursos.

Pesquisas sobre a elevação das temperaturas planetárias associadas às emissões de gases do efeito estufa e ao aumento da frequência e intensidade dos eventos climáticos extremos (tempestades, chuvas extremas, estiagens prolongadas, ondas de calor/frio, por exemplo) são temas de trabalhos produzidos por diversos pesquisadores pelo mundo (VINCENT et al., 2005; HAYLOCK et al., 2006; ALEXANDER et al., 2006; OBREGÓN e MARENGO, 2007; SILLMANN \& ROECKNER 2008; BLAIN, 2010; VALVERDE \& MARENGO, 2014; ALEXANDER, 2016 entre outros).

A análise da particularidade climática de cada região é um fator importante a ser considerado no contexto da ocorrência de eventos pluviométricos extremos. Muito embora existam diversas pesquisas sobre tais eventos, as generalizações devem ser vistas com ressalvas, pois cada lugar possui uma dinâmica peculiar, que nem sempre pode ser explicada por categorias genéricas de um domínio ou tipo climático.

Sant'Anna Neto (2013) discorre sobre a importância da escala regional do clima na compreensão dos climas naturais e nas transformações climáticas produzidas pelos agentes e atores sociais, sendo este o caráter geográfico da análise climática. 
Para Sá Junior (2009) e Novais (2011), o clima de Uberaba (MG) é o do tipo Aw (Tropical de Savana com estação seca de inverno) com temperatura média anual entre $22^{\circ} \mathrm{C}$ e $26^{\circ} \mathrm{C}$ e chuvas anuais entre 1.100 e $1.750 \mathrm{~mm}$, com estação seca entre os meses de maio, junho e julho, sendo considerado como Tropical semiúmido.

Diante da temática dos eventos extremos, a cidade de Uberaba, localizada na região do Triângulo Mineiro, tem sofrido ao longo dos anos com as chuvas intensas e seus desdobramentos. Tal fenômeno (chuvas intensas) associado à maneira pela qual o espaço urbano foi sendo construído (impermeabilização do solo urbano, canalização dos rios e córregos, construção de casas e edifícios em áreas alagáveis etc.) contribuiu para que as eventuais chuvas de maior intensidade provocassem inundações, principalmente nas áreas centrais da cidade.

Enchentes e alagamentos são, historicamente, responsáveis por prejuízos financeiros e perdas humanas, gerando na população um sentimento de insegurança e preocupação durante a estação chuvosa.

Muito embora a população uberabense sofra com a frequente recorrência desses eventos, não existem pesquisas sobre a ocorrência desses valores extremos de chuva ao longo dos anos na cidade.

Atentando pra isto, o presente trabalho tem como objetivo avaliar o comportamento das chuvas locais, identificando os eventos extremos de precipitação, sua frequência e seu tempo de retorno.

\section{MATERIAIS E MÉTODOS}

Para desenvolvimento deste trabalho foram utilizados dados pluviométricos da Estação Meteorológica de Superfície de Uberaba (OMM: 83577) (Figura 1), disponíveis no Banco de Dados Meteorológicos para Ensino e Pesquisa (BDMEP) do Instituto Nacional de Meteorologia (INMET), que se encontra disponível para acesso no seu site. 
Figura 1 - Localização de Uberaba e sua Estação Meteorológica do INMET de Uberaba (OMM: 83577).

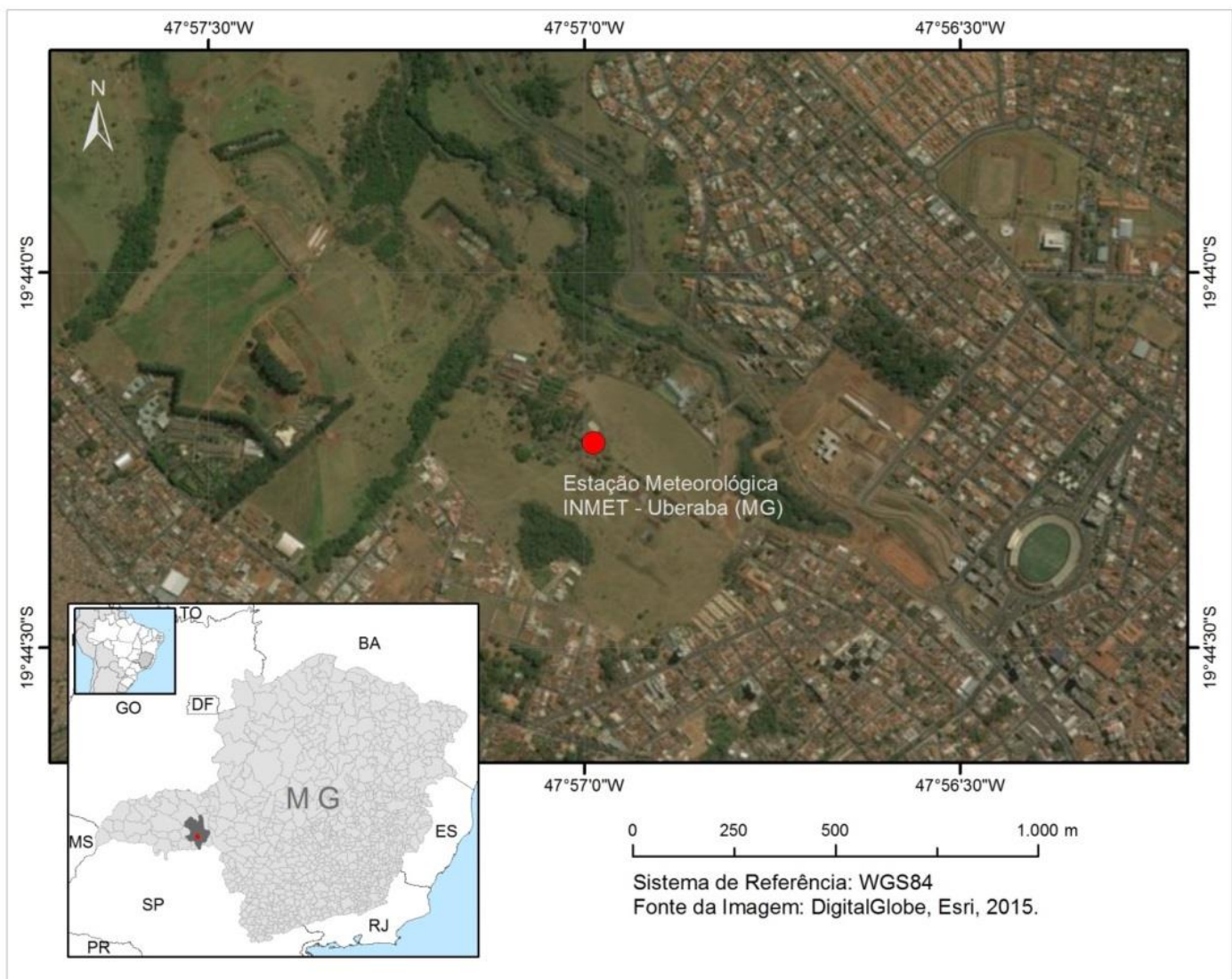

Fonte: autores, 2019.

Foram utilizados dados pluviométricos do período de 1961 a 2015, perfazendo um total de 54 anos. O trabalho de Paz et al (2016) buscou preencher as falhas encontradas na série de dados aplicando-se as técnicas estatísticas (regressão linear e teste dupla-massa) recomendadas Frank et al. (1988); Tucci (2009), Oliveira et al. (2010), Sanches et al. (2012), Sanches (2015).

De posse de uma série temporal de 54 anos, os dados foram organizados em totais mensais para avaliação de suas tendências lineares ao longo da série utilizando o software Excel. Em seguida, a partir dos dados diários, foram extraídos os parâmetros propostos pelo Expert Team on Climate Change Detection and Indices (ETCCDI) para a detecção de evidências de mudanças climáticas (Tabela 1). 
Tabela 1. Parâmetros do ETCCDI para detecção de mudanças climáticas

\begin{tabular}{ll}
\hline Parâmetros ETCCDI & Descrição \\
\hline Rxday & Máximo de chuva acumulada em um único dia no ano \\
Rx5day & Máximo de chuva acumulado em cinco dias no ano \\
R1mm & Dias no mês com chuva igual ou superior a $1 \mathrm{~mm}$ \\
$\mathrm{R} 10 \mathrm{~mm}$ & Dias no mês com chuva igual ou superior a $10 \mathrm{~mm}$ \\
$\mathrm{R} 20 \mathrm{~mm}$ & Dias no mês com chuva igual ou superior a $20 \mathrm{~mm}$ \\
$\mathrm{R} 30 \mathrm{~mm}$ & Dias no mês com chuva igual ou superior a $30 \mathrm{~mm}$ \\
$\mathrm{R} 50 \mathrm{~mm}$ & Dias no mês com chuva igual ou superior a $50 \mathrm{~mm}$ \\
$\mathrm{R} 70 \mathrm{~mm}$ & Dias no mês com chuva igual ou superior a $70 \mathrm{~mm}$ \\
$\mathrm{R} 80 \mathrm{~mm}$ & Dias no mês com chuva igual ou superior a $80 \mathrm{~mm}$ \\
$\mathrm{R} 100 \mathrm{~mm}$ & Dias no mês com chuva igual ou superior a $100 \mathrm{~mm}$ \\
\hline
\end{tabular}

Organizado pelos autores.

Fonte: Traduzido e adaptado de http://etccdi.pacificclimate.org/list_27_indices.shtml

O Teste de Mann-Kendall (MK) foi adotado na avaliação da tendência dos totais pluviométricos mensais e, também, para cada parâmetro recomendado pelo ETCCDI a fim de qualificar as tendências como passíveis, ou não, de mudança de comportamento ao longo da série.

O MK consiste em um teste estatístico não paramétrico recomendado pela Organização Meteorológica Mundial (OMM) e amplamente utilizado para avaliação de tendências climáticas em séries temporais longas (PAIVA e CLARKE, 1995; GROPPO et al., 2001; MARENGO e ALVES, 2005; ALEXANDER et al., 2006; FOLHES e FISCH, 2006; OBREGÓN e MARENGO, 2007; BLAIN, 2010; SANCHES et al., 2013, 2014).

A aplicação do MK foi feita utilizando a ferramenta Mann-Kendall trend test disponível no software PAST (Paleontological statistics software package for education and data analysis) (HAMMER et al., 2001). O PAST é um software estatístico, gratuito, desenvolvido por pesquisadores do Museu de História Natural da Universidade de Oslo, na Noruega.

Nessa avaliação, optou-se pela aplicação do MK com um nível de confiança de 95\% (p-value: 0,05). Para isso, considerou-se que o valor do score $z$ da estatística MK deveria ser $-1,96 \leq \mathrm{z} \leq 1,96$, conforme recomendam Marengo e Alves (2005) e Folhes e Fisch (2006). 
Outro importante parâmetro extraído da série temporal para avaliar a ocorrência de eventos extremos diários de precipitação foi o valor do percentil 99 . O ETCCDI recomenda a identificação do valor do percentil 99 para caracterizar um "evento extremo".

Sendo assim, após a identificação do valor extremo das precipitações diárias por meio do percentil 99, foi extraída a Frequência $(f)$ desse evento ao longo da série, sua Frequência Relativa (fr) (Eq.1) e seu Tempo de Retorno (T) (Eq.2) $c f$. sugerem Galvani (2011) e De Oliveira Sanches et al., (2016).

$$
\begin{array}{ll}
f r=\frac{n a}{n} & \text { Eq. } 1 \\
T=\frac{1}{f r} & \text { Eq. } 2
\end{array}
$$

A frequência absoluta $(f)$ dos eventos que corresponde à quantidade de eventos ocorridos no período estudado; a frequência relativa $(f r)$, que corresponde ao número de vezes que o evento ocorreu $(n a)$ em relação ao número total de elementos da série $(n)$. Seu resultado é expresso em porcentagem. Já o seu Tempo de retorno $(T)$, corresponde o inverso da probabilidade, e estima o retorno desse evento ao longo dos 54 anos da série.

\section{RESULTADOS E DISCUSSÃO}

\section{Análise da tendência dos totais mensais}

O resultado da tendência dos totais pluviométricos mensais para Uberaba pode ser observado na Figura 2. 


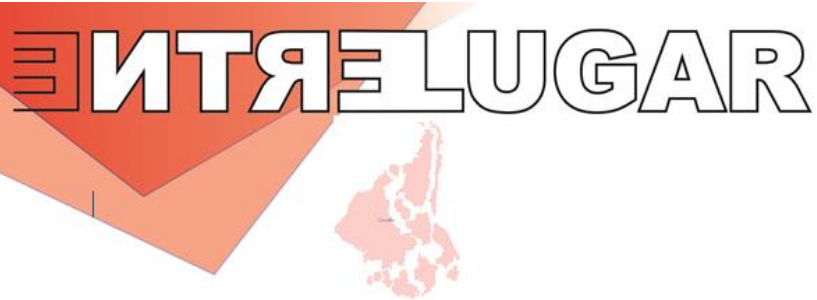

Figura 2 - Tendências dos totais pluviométricos mensais para Uberaba (1961-2015).

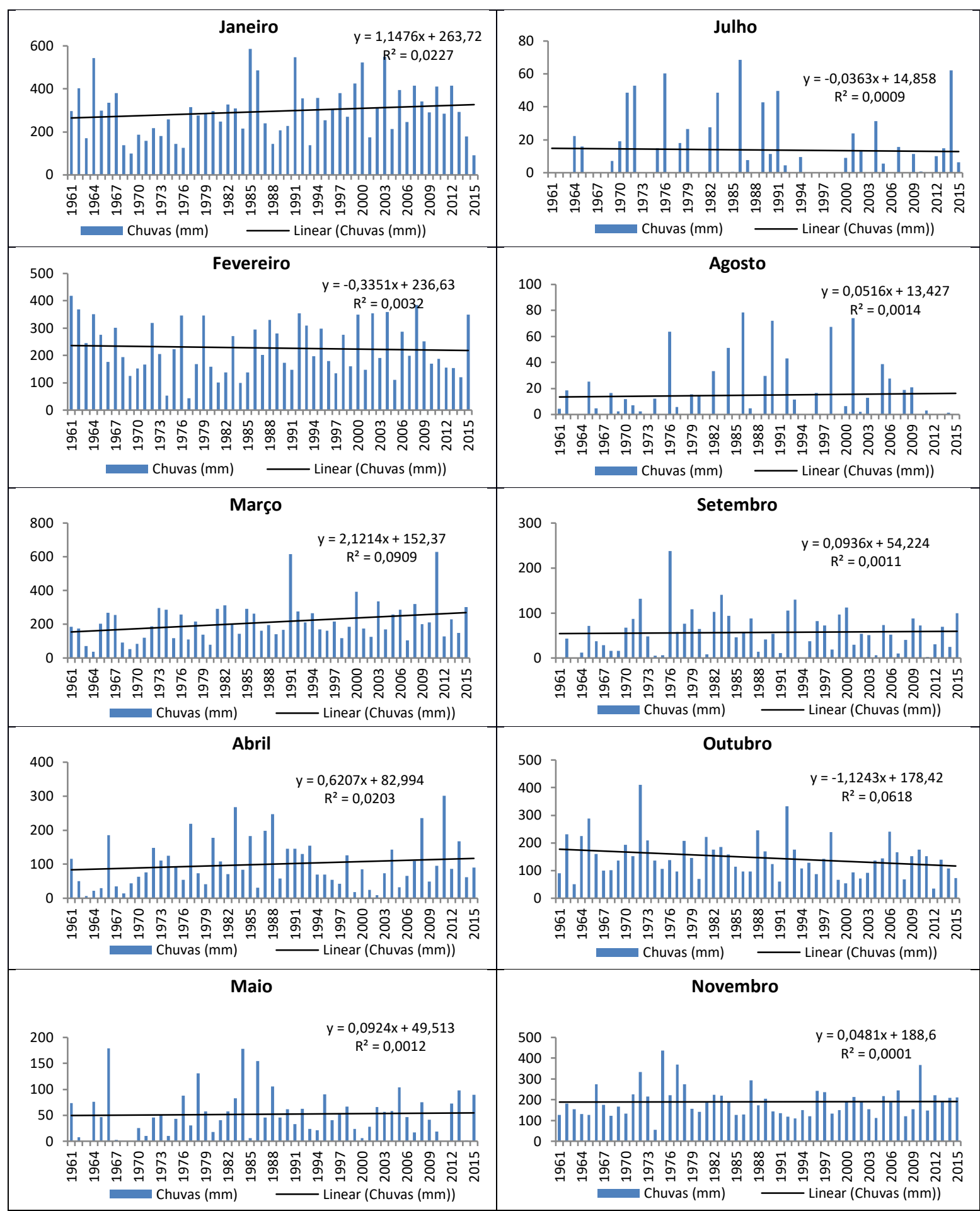

\section{Continua}


Figura 2 (continuação)

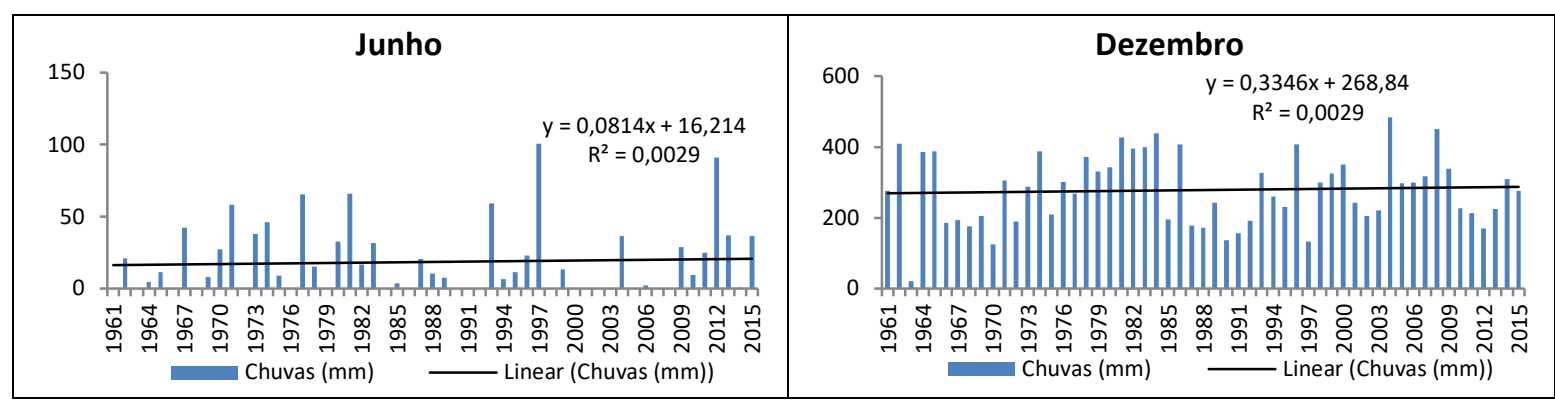

Fonte: autores, 2019..

É possível verificar (Figura 2) que os meses de janeiro, março e abril apresentaram tendência linear positiva com a maior inclinação da reta para a série do mês de março. Nos meses de maio, junho, julho, agosto, setembro, novembro e dezembro a reta da tendência linear observada demonstrou baixo ângulo de inclinação. Já o mês de outubro apresentou tendência linear negativa com um elevado ângulo de sua reta de regressão.

Na Tabela 2 são apresentados os scores $z$, resultado da aplicação da estatística MK para os totais mensais de Uberaba, no período de 1961-2015.

Tabela 2 - Resultado da estatística MK para as tendências lineares dos totais mensais pluviométricos.

\begin{tabular}{lc}
\hline Meses & Score $\boldsymbol{z}$ da estatística MK \\
\hline Janeiro & 0,72 \\
\hline Fevereiro & $-0,30$ \\
\hline Março & $\mathbf{2 , 0 0 *}$ \\
\hline Abril & 1,26 \\
\hline Maio & 1,11 \\
\hline Junho & $-0,18$ \\
\hline Julho & 0,77 \\
\hline Agosto & $-0,23$ \\
\hline Setembro & 0,89 \\
\hline Outubro & $\mathbf{- 1 , 9 6 *}$ \\
\hline Novembro & $-0,19$ \\
\hline Dezembro & $-0,06$ \\
\hline
\end{tabular}

*Valores considerados significativos para mudança de comportamento após a estatística MK.

Fonte: autores, 2019. 
Os resultados (Tabela 2) demonstram que a tendência de aumento nos totais mensais para o mês de março foi considerada significativa (score $z=2,00$ ) para mudança de comportamento após a aplicação do MK.

Considerando-se que a Normal Climatológica (1961-1990) para as chuvas no mês de março, em Uberaba, é de 186,90 mm, a ocorrência de totais pluviométricos mensais superiores a $600 \mathrm{~mm}$ nos anos de 1991 e 2001, e superiores a $300 \mathrm{~mm}$ nos anos de 2000, 2003, 2008 e 2015, demonstram um aumento na frequência de meses chuvosos, sobretudo a partir da última década do século XX.

Cabe ressaltar que dos anos acima destacados (1991, 2000, 2003, 2008, 2011 e 2015), os anos de 2000, 2008 e 2011 foram anos sob efeito da componente negativa (La Niña) do fenômeno El Niño Oscilação Sul (ENOS). Já os anos de 1991, 2003 e 2015 foram considerados neutros pelo monitoramento das águas do Pacífico (NOAA, 2016). Dessa forma, não é possível relacionar os eventos intensos de pluviosidade mensal para os meses de março com a participação do fenômeno ENOS.

A Figura 2 revela, ainda, a tendência negativa observada para o mês de outubro, a qual foi considerada pelo MK como significativa para mudança de comportamento uma vez que seu score $z=-1,96$ apresentou resultados fora do intervalo de confiança, em um nível de $95 \%$.

De acordo com informações obtidas no site do INMET, a Normal Climatológica 1961-1990 para a pluviosidade do mês de outubro, em Uberaba, é de 167,8 mm. O gráfico das chuvas mensais de outubro (Figura 2) mostra que, a partir dos anos 1990, ocorreram 10 anos em que as chuvas não ultrapassaram o valor de $100 \mathrm{~mm}$ para o referido mês.

Segundo informações sobre o monitoramento do ENOS (NOAA, 2016), a partir de 1990, apenas os anos de 1999 e 2000 foram influenciados pela componente negativa (La Niña). Os anos de 1991, 2002 e 2015 foram influenciados pela fase positiva do fenômeno ENOS (El Niño). Já os demais anos com chuvas mensais de outubro inferiores a $100 \mathrm{~mm}$ foram considerados como anos neutros. Sendo assim, é possível 


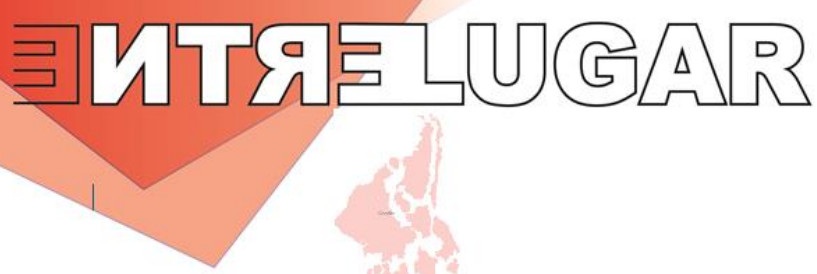

descartar a influência direta do principal fenômeno oceânico-atmosférico responsável pelas anomalias pluviométricas na região.

\section{Análise dos parâmetros do ETCCDI}

O resultado da aplicação da estatística MK aos parâmetros propostos pelo ETCCDI pode ser observado na Tabela 3.

Tabela 3 - Resultado do score (z) da estatística Mann-Kendall para os parâmetros do ETCCDI

\begin{tabular}{lllllllllllll}
\hline & Jan & Fev & Mar & Abr & Mai & Jun & Jul & Ago & Set & Out & Nov & Dez \\
\hline Rxday & 0,62 & 0,04 & 0,67 & 0,49 & 1,47 & $-0,74$ & $-0,49$ & 0,02 & 1,20 & $-0,57$ & $-0,46$ & 0,92 \\
Rx5day & 1,78 & $-0,57$ & 1,79 & 1,39 & 1,73 & $-0,74$ & 0,63 & 0,02 & 1,09 & $-1,33$ & $-0,81$ & 0,37 \\
R1mm & $-0,23$ & 0,18 & $\mathbf{2 , 5 0}$ & 1,57 & 0,81 & $-0,76$ & $-0,03$ & $-0,56$ & 0,41 & $-2,73$ & 0,98 & 0,62 \\
R10mm & 1,00 & 0,23 & 1,75 & 1,66 & 1,49 & 0,12 & 0,29 & 0,00 & 0,45 & $-1,95$ & $-0,11$ & $-0,32$ \\
R20mm & 1,34 & $-0,05$ & 1,06 & 0,86 & 1,21 & 0,34 & $-0,17$ & 1,12 & $-0,35$ & $-1,38$ & $-0,42$ & $-1,21$ \\
R30mm & 1,52 & $-0,27$ & 0,47 & 0,67 & $\mathbf{2 , 4 7}$ & 0,46 & $-0,55$ & 1,2 & 0,55 & $-0,17$ & $-0,76$ & 0,37 \\
R50mm & 0,5 & $-0,57$ & 0,77 & $-0,35$ & $-0,04$ & 0,53 & 0 & 0 & 0,36 & $-0,69$ & $-0,34$ & 0,94 \\
R70mm & 1,13 & $-0,17$ & $\mathbf{2 , 2 1}$ & $-0,08$ & $-0,26$ & 0 & 0 & 0 & 0 & $-0,32$ & $-0,46$ & 0,46 \\
R80mm & 1,19 & 1,22 & 1,16 & $-1,20$ & $-0,20$ & 0 & 0 & 0 & 0 & $-0,72$ & $-1,40$ & 0,37 \\
R100mm & $-0,21$ & 1,22 & 0,21 & $-0,58$ & 0 & 0 & 0 & 0 & 0 & $-0,37$ & $-1,61$ & 0 \\
\hline
\end{tabular}

Fonte: autores, 2019.

As informações da estatística MK (Tabela 3) demonstram que, para o mês de março, a quantidade de dias de chuva (R1mm) e os dias com chuvas superiores a $70 \mathrm{~mm}$ (R70mm) apresentaram scores com significância estatística, ou seja, seus scores mostraram-se fora do intervalo de confiança, em um nível de significância de $95 \%$ (pvalue: 0,05). A Figura demonstra a tendência de aumento nos dias na quantidade de dias com R1mm (Figura 3a) e dias com R70mm (Figura 3b).

Esses resultados corroboram com os resultados indicados pelo MK para os totais mensais em março, demonstrando que além de maiores volumes mensais, o mês de março tem apresentado mais dias chuvosos e dias com chuva mais intensa.

Figura 3 - Tendências lineares do R1mm (a) e R70mm (b) para o mês de março

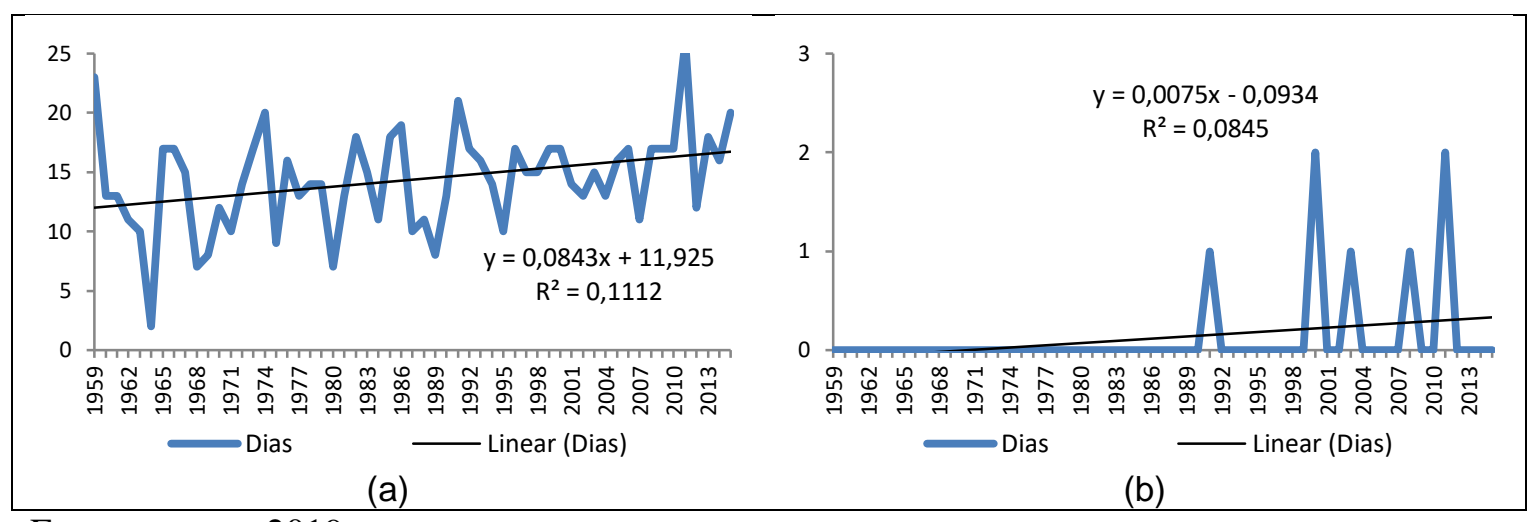

Fonte: autores, 2019. 
A tendência positiva dos dias com chuvas superiores a $30 \mathrm{~mm}$ (R30) no mês de maio (Figura 4a) também foi considerada significativa pelo MK para mudança de comportamento $(\mathrm{z}=2,21)$.

Figura 4 - Tendência linear do R30mm para o mês de maio (a) e dos dias com chuva (chuvas R1mm) para o mês de outubro (b)

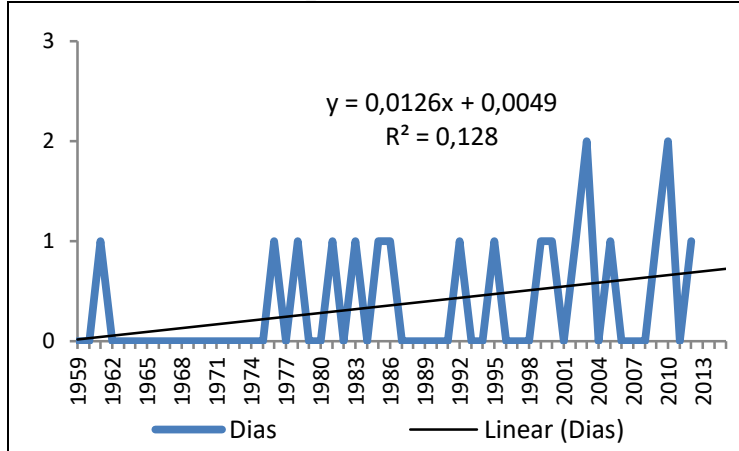

(a)

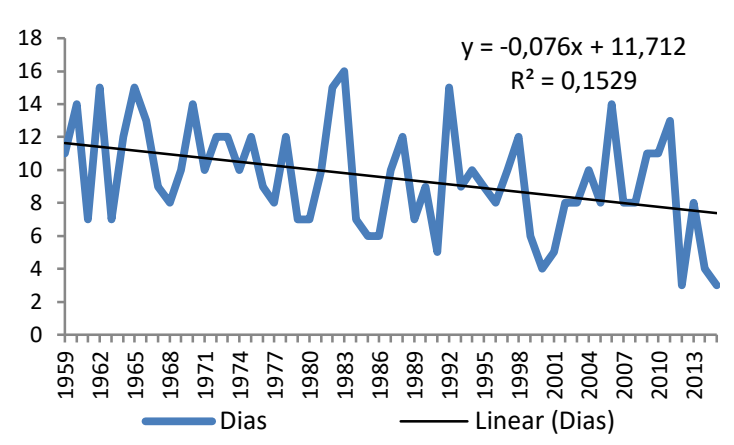

(b)

Fonte: autores, 2019.

Por outro lado, considerando a quantidade de dias com chuva (R1mm), o mês de outubro (Figura 4b) apresentou tendência negativa considerada significativa pelo MK $(z=-2,73)$. Dessa forma, os dados sugerem que, no mês de outubro, esteja ocorrendo menos episódios de chuva diária.

A redução na quantidade de dias de chuva para o mês de outubro, em Uberaba, corrobora com os resultados observados na tendência dos totais mensais (Figura 2), os quais demonstram redução significativa nos valores pluviométricos para o mês de outubro.

Dessa forma, os resultados apontados pelo MK para os dias com chuva no mês de outubro (R1mm), associado aos resultados encontrados na tendência dos totais mensais sugerem, com maior evidência estatística, que haja uma tendência de prolongamento da estação seca (dias sem chuva) na região de Uberaba e, que tal comportamento, pode ser considerado como evidência de mudanças climáticas em escala local.

Para Sanches et al (2017) esse prolongamento da estação seca está associado a atuação de bloqueios atmosféricos devido a alterações no Sistema de Monções da 
América do Sul (SMAS), como um dos efeitos do aquecimento global, como fundamenta o trabalho de Pascale et al. (2019).

\section{Análise dos eventos extremos de precipitação diária}

Outro parâmetro recomendado pelo ETCCDI para a identificação de eventos extremos é o valor do percentil 99.

A partir dos dados pluviométricos diários analisados no período de 19612015, a estatística do percentil 99 indicou como valor extremo de precipitação chuvas diárias superiores a 52,4 mm. No entanto, ao observar a Figura 5 verifica-se que valores diários de precipitação são muito comuns. Dessa forma, optou-se por segmentar os valores pluviométricos diários superiores ao indicado pelo percentil 99 e, assim, calcular sua frequência, frequência relativa e seu tempo de retorno.

A segmentação considerou os eventos pluviométricos nos intervalos entre 52,4 $\mathrm{mm}$ e $60 \mathrm{~mm}, 60 \mathrm{~mm}$ e $80 \mathrm{~mm}, 80 \mathrm{~mm}$ e $100 \mathrm{~mm}, 100 \mathrm{~mm}$ e $120 \mathrm{~mm}$ e acima de 120 $\mathrm{mm}$.

A partir dessas informações foi possível estabelecer o comportamento dos dados em relação a sua ocorrência identificando se tais eventos estão se tornando ou não mais frequentes e intensos.

A Tabela 4 apresenta os resultados dos parâmetros analisados (frequência, frequência relativa e tempo de retorno).

Tabela 4 - Cálculo da Frequência, Frequência Relativa e do Tempo de Retorno dos eventos intensos de precipitação para a série Uberaba (1961-2015)

\begin{tabular}{lccc}
\hline Eventos & Frequência & $\begin{array}{c}\text { Freq. Relativa } \\
(\boldsymbol{\%})\end{array}$ & $\begin{array}{l}\text { Tempo de Retorno em } \\
\text { anos }\end{array}$ \\
\hline$>120 \mathrm{~mm}$ & 4 & $6,9 \%$ & 15 \\
$100-120 \mathrm{~mm}$ & 8 & $13,8 \%$ & 7 \\
$80-100 \mathrm{~mm}$ & 27 & $46,6 \%$ & 2 \\
$60-80 \mathrm{~mm}$ & 90 & $155,2 \%$ & 0,6 (a cada oito meses) \\
$52,4-60 \mathrm{~mm}$ & 77 & $132,8 \%$ & 0,8 (a cada nove meses) \\
\hline
\end{tabular}

Fonte: autores, 2019.

Os resultados revelaram que eventos pluviométricos diários entre $52,4 \mathrm{~mm}$ (percentil 99) e $60 \mathrm{~mm}$, e entre $60 \mathrm{~mm}$ e $80 \mathrm{~mm}$ apresentam uma elevada probabilidade 
de ocorrência, podendo ocorrer mais de uma vez ao ano. A estimativa de seu tempo de retorno médio é de nove e oito meses, respectivamente.

Caso um evento dessa intensidade ocorra nos meses de janeiro, fevereiro e março, por exemplo, há uma grande probabilidade que até o final deste ano outro evento dessa intensidade venha a ocorrer.

Os eventos pluviométricos entre $80 \mathrm{~mm}$ e $100 \mathrm{~mm}$, por sua vez, possuem uma frequência menor ( 27 eventos ao longo da série) e com um tempo de retorno médio de dois anos. Os eventos diários entre $100 \mathrm{~mm}$ e $120 \mathrm{~mm}$ possuem uma frequência ainda menor, retornando, pelo menos, a cada sete anos.

Por fim, ao longo da série analisada, ocorreram apenas quatro eventos pluviométricos superiores a $120 \mathrm{~mm} /$ dia. Tais eventos apresentam uma baixa probabilidade de ocorrência (frequência relativa) e um tempo médio de retorno longo (15 anos).

No entanto, eventos pluviométricos acima de 52,4 mm (valor do percentil 99) costumam promover profundos problemas aos munícipes. Exemplos dos transtornos causados pelas enchentes e alagamentos podem ser vistos (Figura 6) ao longo de duas das principais avenidas da cidade: Av. Leopoldino de Oliveira (sentido oeste- leste) (Figura 6a), Av. Guilherme Ferreira (sentido norte-sul) (Figura 6d) e a Av. Santos Dumont (sentido norte-sul) (Figuras 6b; 6c).

Por localizarem-se nas áreas mais baixas da cidade e possuírem rios e córregos canalizados, o escoamento das águas pluviais promove, de forma rápida, a elevação das águas culminando no alagamento dessas avenidas. 
Figura 5 Sequência de dados pluviométricos diários para Uberaba (1961-2015).

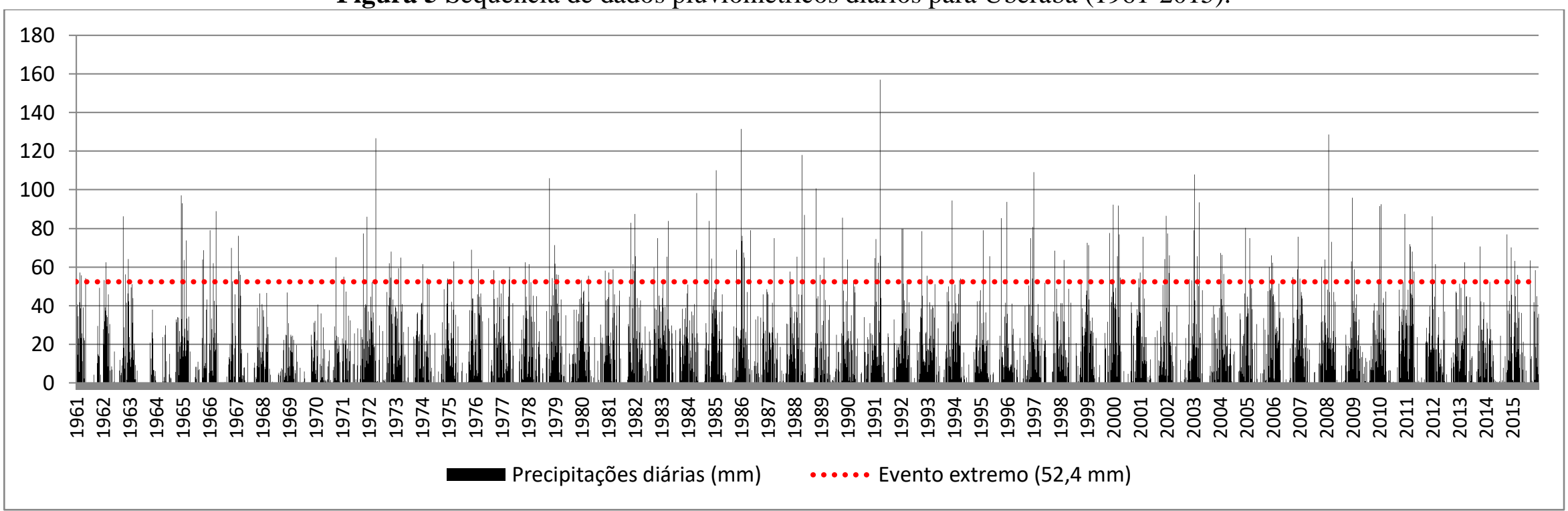

Elaborado pelo autores.

Fonte: Dados do BDMEP/INMET, organizado pelos autores. 
Figura 6 - Imagens de enchentes e alagamentos na região central da cidade de Uberaba. Av. Leopoldino de Oliveira (a), encontro das avenidas Santos Dumont com a Leopoldino de Oliveira (b), Av. Santos Dumont (c) e Av. Guilherme Ferreira (d).

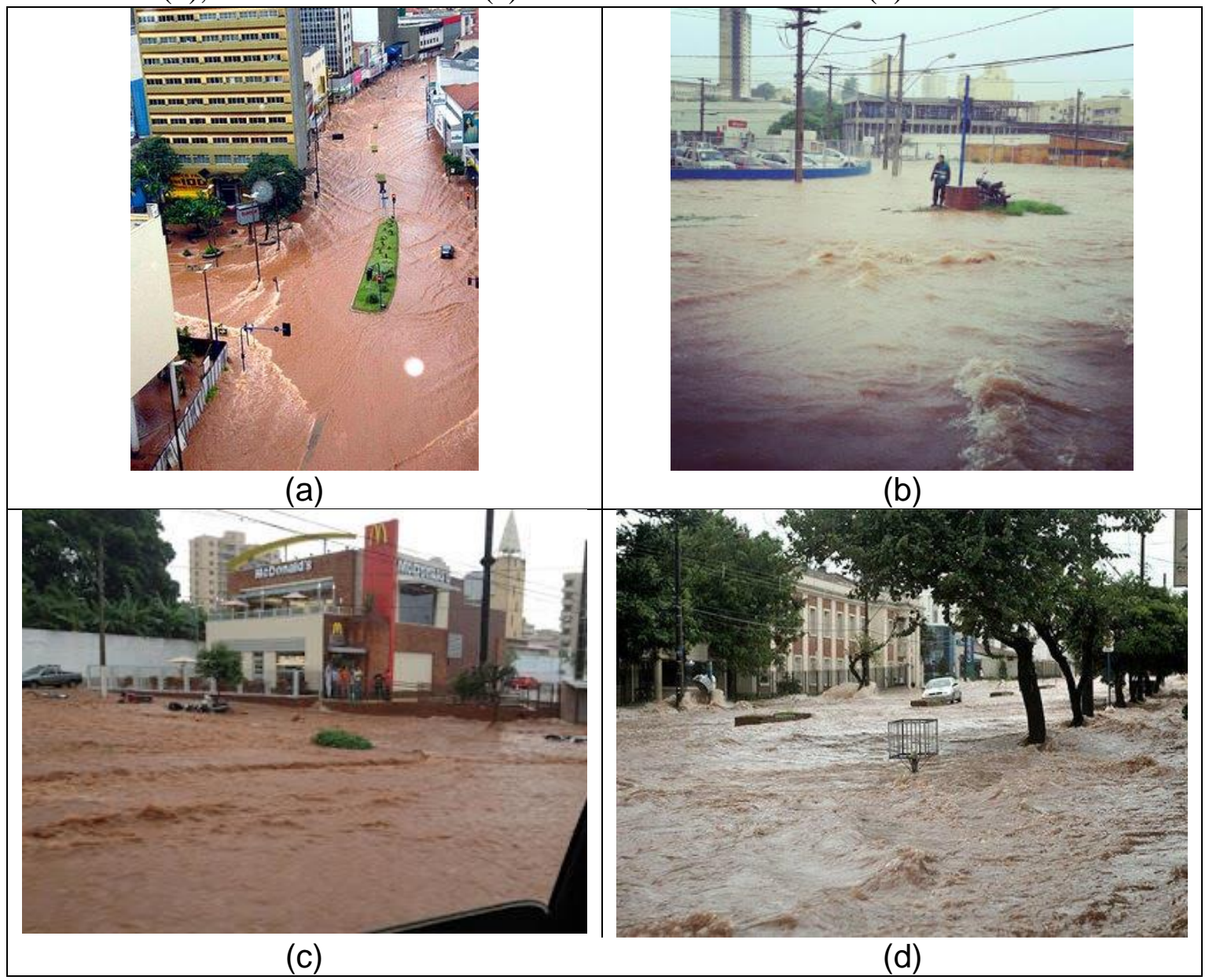

Organizado pelos autores.

Fonte: Adaptado de http://omelhordeuberaba.blogspot.com.br/

\section{CONCLUSÕES}

Muito embora episódios de enchentes tenham sido recorrentes na cidade de Uberaba, sobretudo a partir da segunda metade do século XX, a inexistência de estudos que abordassem o tema levou a necessidade de se identificar os eventos pluviométricos de grande intensidade (eventos extremos), sua frequência e estimar o período de retorno destes.

Para isso, buscou-se aplicar um conjunto de técnicas (em sua maioria estatística) visando à identificação de tais parâmetros. 
Verificou-se que os eventos chuva no mês de março demonstraram ter se tornado mais intensos e mais frequentes a partir do final do século $\mathrm{XX}$, e que, esse aumento, considerado significativo a partir de avaliação estatística, pode ser considerado como evidência de mudanças climáticas como sugere a literatura fundamentada (VINCENT et al., 2005; ALEXANDER et al., 2006; SILLMANN \& ROECKNER 2008; VALVERDE \& MARENGO, 2014; ALEXANDER, 2016 entre outros).

A intensificação dos eventos pluviométricos extremos pode trazer sérios prejuízos aos munícipes uma vez que, ao ocorrerem sobre as áreas centrais da cidade, o intenso processo de impermeabilização do solo urbano, peculiar à maior parte das cidades brasileiras, associada à canalização de rios e córregos urbanos contribuem para ocorrência de enchentes e alagamentos nas áreas mais baixas da cidade. Armond e Sant'Anna Neto (2017) consideram serem esses, entre outros, os fatores geográficos do clima.

Da mesma forma, ao se analisar as chuvas no mês de outubro, verifica-se que além da redução na quantidade de dias com chuva, há uma tendência de redução nos totais pluviométricos mensais. Acredita-se que essa redução nas precipitações esteja associada à tendência de prolongamento da estação seca pela atuação de bloqueios atmosféricos $c f$. salienta Sanches et al (2017).

Dessa forma, a partir da análise dos dados e segundo a metodologia e técnicas propostas para o estudo, foi possível constatar evidências de mudanças no comportamento das chuvas passíveis de serem consideradas como decorrentes das mudanças climáticas.

Considerando que a empresa de abastecimento público - CODAU Companhia Operacional de Desenvolvimento, Saneamento e Ações Urbanas - capta água diretamente do rio Uberaba, a aproximadamente $4 \mathrm{~km}$ do centro da cidade, o prolongamento do período de estiagem contribui para o rebaixamento do curso d'água, levando a possibilidade de racionamento de água à população entre os meses de setembro e outubro.

Dessa forma, espera-se que os resultados obtidos neste trabalho sobre o comportamento das chuvas em Uberaba nos últimos 54 anos possam subsidiar o poder 


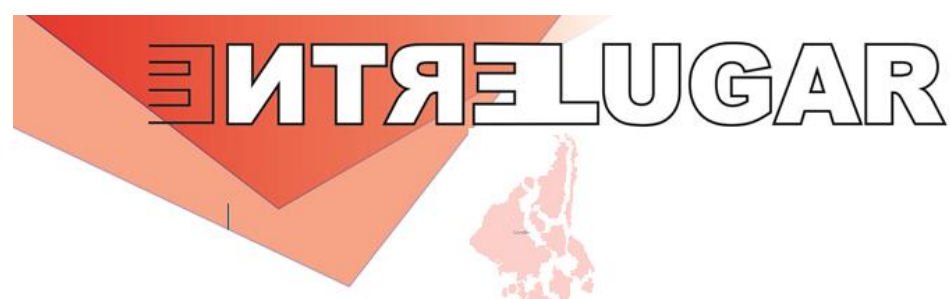

público municipal, a Defesa Civil e demais órgãos, nas ações de planejamento, gestão e mitigação dos problemas decorrentes, ora do excesso, ora da escassez de chuvas.

\section{REFERÊNCIAS}

ALEXANDER, L.V; ZHANG, X.; PETERSON, T.C.; CAESAR, J.; GLEASON, B.; KLEIN TANK, A.M.G.; HAYLOCK, M.; COLLINS, D.; TREWIN, B.; RAHIMZADEH, F.; TAGIPOUR, A.; RUPA KUMAR, K.; REVADEKAR, J.; GRIFFITHS, G.; VICENT, L.; STEPHENSON, D. B.; BURN, J.; AGUILAR, E.; TAYLOR, M.; NEW, M.; ZHAIN, P.; RUSTICUCCI, M.; VAZQUEZ-AGUIRRE, J.L. Global observed changes in daily climate extremes of temperature and precipitation. Journal of Geophysical Research. vol. 111, D05109, 2006. (doi: 10.1029/2005JD00690).

ALEXANDER, L.V. Global observed long-term changes in temperature and precipitation extremes: A review of progress and limitations in IPCC assessments and beyond. Weather and Climate Extremes. v.11, p.4-16. 2016. (doi:10.1016/j.wace.2015.10.007)

ARMOND, N.B.; SANT'ANNA NETO, J.L. Entre eventos e episódios: ritmo climático e excepcionalidades para uma abordagem geográfica do clima no município do Rio de Janeiro. Revista Brasileira de Climatologia, ano 13, v. 20, jan/jul, p.5-28, 2017.

BLAIN, G.C. Detecção de tendências monótonas em séries mensais de precipitação pluvial no Estado de São Paulo. Bragantia, Campinas, v.69, n4, p.1027-1033, 2010.

DE OLIVEIRA SANCHES, F.; VERDUM, R.; FISCH, G. Chuvas extremas em Alegrete (RS): um estudo sobre sua ocorrência no Século XX. Boletim Goiano de Geografia, [S.1.], v. 36, n. 2, p. 340, ago. 2016.

ETCCDI/CRD Climate Change. Disponível em: http://etccdi.pacificclimate.org/list_27_indices.shtml Acesso em: 15 fevereiro 2020.

FOLHES, M.T.; FISCH, G. Caracterização climática e estudo de tendência nas séries temporais de temperatura do ar e precipitação em Taubaté (SP). Ambi-Agua, Taubaté, v.1, n.1, p.61-71, 2006.

FRANK, B.; SEVERO, D.; SILVA, H.S. Validade do preenchimento de falhas em séries temporais. In: V Congresso Brasileiro de Meteorologia. Anais... Rio de Janeiro, 1988.

GALVANI, E. Estatística descritiva em sala de aula. In: VENTURI, L.A.B. Geografia: práticas de campo, laboratório e sala de aula. São Paulo: Ed. Sarandi, 2011.

GROPPO, J.D.; MILDE, L.C.E.; GUAMERO, M.E.; MORAES, J.M.; MARTINELLI, L.A. Análise de séries temporais de vazão e de precipitação na Bacia do Rio Piracicaba. Revista de Ciência \& Tecnologia. v.8, n.18, p.109-117, 2001.

HAMMER, Ø.; HARPER, D.A.T.; RYAN, P.D. PAST: Paleontological statistics software package for education and data analysis. Palaeontologia Electronica 4(1): 2001. 9pp

HAYLOCK, M.R.; PETERSON, T.C.; ALVES, L.M.; AMBRIZZI, T.; ANUNCIAÇÃO, Y.M.T.; BAEZ, J.; BARROS, V.R; BERLATO, M.A.; BIDEGAIN, M.; CORONEL, G.; 


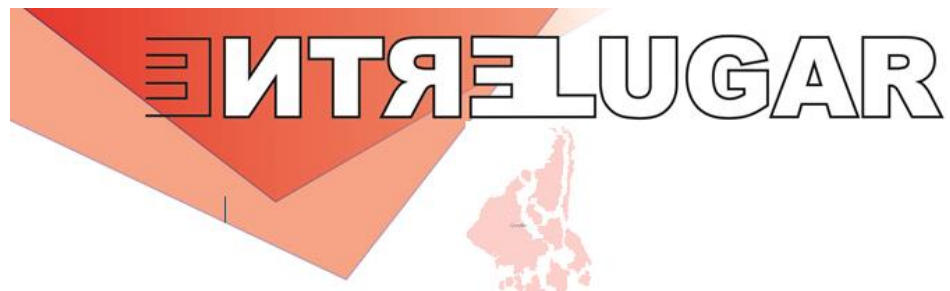

CORRADI, V.; GARCIA, J.; GRIMM, A.M.; KAROLY, D.; MARENGO, J.A.; MARINO, M.B.; MONCUNILL, D.F.; NECHET, D.; QUINTANA, J.; REBELLO, E.; RUSTICUCCI, M.; SANTOS, J.L.; TREBEJO, I.; VINCENT, L.A. Trends in Total and Extreme South American Rainfall in 1960-2000 and Links with Sea Surface Temperature. Journal of Climate, v.19, p.1490-1512, 2006.

INMET. Disponível em: http://www.inmet.gov.br/portal/. Acesso em: 15 fevereiro 2020.

INMET/BDMEP.

Disponível

em: http://www.inmet.gov.br/portal/index.php?r=bdmep/bdmep

IPCC - Intergovernmental Panel on Climate Change: Climate Change 2014: Synthesis Report. Contribution of Working Groups I, II and III to the Fifth Assessment Report of the Intergovernmental Panel on Climate Change. IPCC, Geneva, Switzerland, 2014. $151 \mathrm{p}$.

MARENGO, J.A.; ALVES, L.M. Tendências hidrológicas da bacia do rio Paraíba do Sul. Revista Brasileira de Meteorologia, v.20, n.2, 215-226, 2005.

MENDONÇA, F.; DANNI-OLIVEIRA, I.M. Climatologia: noções básicas e climas do Brasil. São Paulo: Oficina de Textos, 2007.

National Oceanic and Atmospheric Administration (NOAA). National Weather Service: Climate Prediction Center. El Niño Southern Oscillation, 2016. http://www.cpc.ncep.noaa.gov/products/analysis_monitoring/ensostuff/ensoyears.shtml >. Acesso em 01 de março de 2016.

NOVAIS, G.T. Caracterização climática da mesoregião do Triângulo Mineiro/Alto Paranaíba e do entorno da Serra da Canastra (MG). 175f. Dissertação (Mestrado em Geografia) - Universidade Federal de Uberlândia, UFU, Uberlândia, 2011.

OBREGÓN, G.; MARENGO, J.A. Caracterização do clima no Século XX no Brasil: Tendências de chuvas e Temperaturas Médias Extremas. Relatório $n^{\circ} 2$. Ministério do Meio Ambiente. Secretaria de Biodiversidade e Florestas. Diretoria de Conservação da Biodiversidade. 2007.

O Melhor de Uberaba (Blog): http://omelhordeuberaba.blogspot.com.br. Acesso: 16 fev 2020.

OLIVEIRA, L.F.C.; FIORENZE, A.P.; MEDEIROS, A.M.M.; SILVA, M.A.S. Comparação de metodologias de preenchimento de falhas de séries históricas de precipitação pluvial anual. Revista Brasileira de Engenharia Agrícola e Ambiental. v.14, n.11, p.1186-1192, 2010.

PAIVA, E.M.C.D.; CLARKE, R.T. Análise de tendência de precipitação na Amazônia. Revista Brasileira de Meteorologia. n.10 (1/2), p.37-41, 1995.

PASCALE, S.; CARVALHO, L.M.V.; ADAMS, D.K.; CASTRO, C.L.; CAVALCANTI, I.F.A. Current and Future Variations of the Monsoons of the Americas in a Warming Climate. Curr Clim Change Rep, 5, 125-144, 2019.

PAZ, C.M.V.; SANCHES, F.; FERREIRA, R.V.; MACHADO, V. Tendência das chuvas mensais para Uberaba (1961-2015). In: XII Simpósio Brasileiro de Climatologia Geográfica. Anais... Goiânia-GO, 2016. p. 2617-2628. 


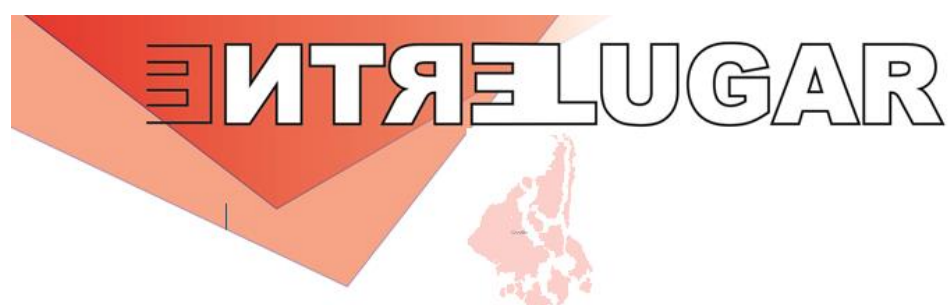

SÁ JUNIOR, A. Aplicação da classificação de Köppen para o Zoneamento Climático do Estado de Minas Gerais. 101f. Dissertação (Mestrado) Universidade Federal de Lavras (UFV), Lavras, 2009.

SANCHES, F.O. O Geógrafo-Climatologista e as mudanças climáticas: uma proposta metodológica. Revista Equador, v. 4, p. 101-118, 2015.

SANCHES, F.; SILVA, R.V.; FERREIRA, R.V.; CAMPOS, C.A.A. Climate change in the Triângulo Mineiro region - Brazil. Revista Brasileira de Climatologia, ano 13, v.21, jul/dez, p. 570-587, 2017.

SANCHES, F.O.; VERDUM, R.; FISCH, G. Preenchimento de falhas em série de dados pluviométricos de Uruguaiana (RS) e análise de tendência. In: XVII Congresso Brasileiro de Meteorologia. Anais... Gramado-RS. 2012.

SANCHES, F.O.; VERDUM, R.; FISCH, G. Trend analysis of long-term rainfall. Revista Ambiente \& Água, v. 8, p. 212-228, 2013.

SANCHES, F.O.; VERDUM, R.; FISCH, G. Tendência de longo prazo das chuvas diárias no Sudoeste do Rio Grande do Sul: os eventos extremos e a arenização. Revista Brasileira de Geografia Física, v. 7, p. 1100-1109, 2014.

SANT'ANNA NETO, J.L. Escalas geográficas do clima: mudança, variabilidade e ritmo. In: AMORIM, M.C.D.C.T.; NETO, J.L.S.; MONTEIRO, A. Climatologia Urbana e Regional: questões teóricas e estudos de caso. São Paulo: Outras Expressões, 2013.

SILLMANN, J.; ROECKNER, E. Indices for extreme events in projections of anthropogenic climate change. Climatic Change. 86:83-104, 2008.

TUCCI, C.E.M.(Org.) Hidrologia: ciência de aplicação. 4.ed., $1^{a}$ reimp. - Porto Alegre: Editora da UFRGS/ABRH, 2009.

VALVERDE, M.C.; MARENGO, J.A. Extreme rainfall indices in the hidrographic basins of Brazil. Open Journal of Modern Hidrology, v.4, n.1, p. 10-26. 2014.

VINCENT, L.A.; PETERSON, T.C.; BARROS, V.R; MARINO, M.B.; RUSTICUCCI, M.; CARRASCO, G.; RAMIREZ, E.; ALVES, L.M.; AMBRIZZI, T.; BERLATO, M.A.; GRIMM, A.M.; MARENGO, J.A.; MOLION, L.; MONCUNILL, D.F.; REBELLO, E.; ANUNCIAÇÃO, Y.M.T.; QUINTANA, J.; SANTOS, J.L.; BAEZ, J.; CORONEL, G.; GARCIA, J.; TREBEJO, I.; BIDEGAIN, M.; HAYLOCK, M.R.; KAROLY, D. Observed Trends in Indices of Daily Temperature Extremes in South America 19602000. Journal of Climate, v.18, p.5011-5023, 2005.

\section{AGRADECIMENTOS}

Agradecimentos à Pró-reitoria de Pesquisa e Pós-graduação da Universidade Federal do Triângulo Mineiro (PROPPG/UFTM), bem como a Fundação de Amparo à Pesquisa do Estado de Minas Gerais (FAPEMIG) pelo auxílio financeiro concedido.

Recebido para publicação em agosto de 2019. Aceito para publicação em fevereiro de 2020. 